\title{
Additional Midline Biopsies of the Peripheral Zone Associated with the First Endorectal Standard Sextant Pattern Improves the Accuracy of Prostate Cancer Detection in Japanese Patients
}

\author{
Norihito Soga $^{a} \quad$ Yasushi Yatabe $^{b} \quad$ Yuji Ogura ${ }^{a} \quad$ Norio Hayashi $^{a}$ \\ aDepartment of Urology and bepartment of Pathology, Aichi Cancer Center Hospital, Nagoya, Japan
}

\author{
Key Words \\ Additional midline • Diagnosis accuracy • Prostate cancer • \\ First endorectal biopsy $\cdot$ Systematic biopsy
}

\begin{abstract}
Objectives: This study was designed to estimate the improved accuracy of prostate cancer ( $\mathrm{PCa}$ ) detection resulting from additional midline biopsies of the peripheral zone in first standard biopsy. Patients and Methods: Patients were classified into 3 groups: 402 cases of sextant biopsies (19952002), 488 cases of 8-core biopsies with 2 additional midline biopsies (2003-2006), and 391 cases of 10-core biopsies with 4 additional midline biopsies (2007-2012). The positive rate of each number of biopsies and changes in positive rates associated with prostate specific antigen (PSA) ranges were estimated. Results: The positive rate of core biopsy significantly improved with increasing numbers of core biopsies (30.1\% for sextant, $43.4 \%$ for 8 -core biopsies, and $53.1 \%$ for 10-core biopsies). The accuracy of biopsies for each PSA range also significantly improved $(22.3 \%$ for sextant, $30.0 \%$ for 8 -core biopsies, and $43.2 \%$ for 10 -core biopsies in the PSA gray zone $[4.01-10 \mathrm{ng} / \mathrm{ml}]$; and $26.5 \%$ for sextant, $52.9 \%$ for 8 -core biopsies, and $71.8 \%$ for 10 -core biopsies in the intermediate PSA range [10.1-20 $\mathrm{ng} / \mathrm{ml}])$. In the 208 cases with positive results using the 10-core biopsy method, the distribution of Gleason scores did not differ between the sextant
\end{abstract}

\section{KARGER}

Fax +4161306 1234

E-Mail karger@karger.ch

www.karger.com
(C) 2014 S. Karger AG, Basel

1015-9770/14/0082-0074\$38.00/0

Accessible online at:

www.karger.com/cur only group and the midline site only group. Conclusions: Additional midline biopsy was associated with improved accuracy of positive core biopsies in Japanese patients with a PSA range of $4.01-20 \mathrm{ng} / \mathrm{ml}$.

Copyright $\odot 2014$ S. Karger AG, Basel

\section{Introduction}

Random, systematic, ultrasound guided transrectal 6-core biopsies of the prostate to detect the prostate cancer $(\mathrm{PCa})$ has been the standard biopsy method for several decades [1]. However, an optimal method using additional biopsies has been recently discussed, particularly with respect to their association with elevated prostate specific antigen (PSA) levels and positive digital examination, and the majority of methods proposed focus on the benefit of additional lateral biopsy, as a 19-24\% improvement of detection rates was reported in several studies [2-4]. In Western countries, the midline portion of the biopsy contributes no additional advantage with respect to cancer detection, but systematic 12-cores biopsies, including laterally directed cores to the standard sextant schema, is strongly recommended [5, 6]. Although this protocol was established using Western populations [7], it is unknown whether these recommendations can 


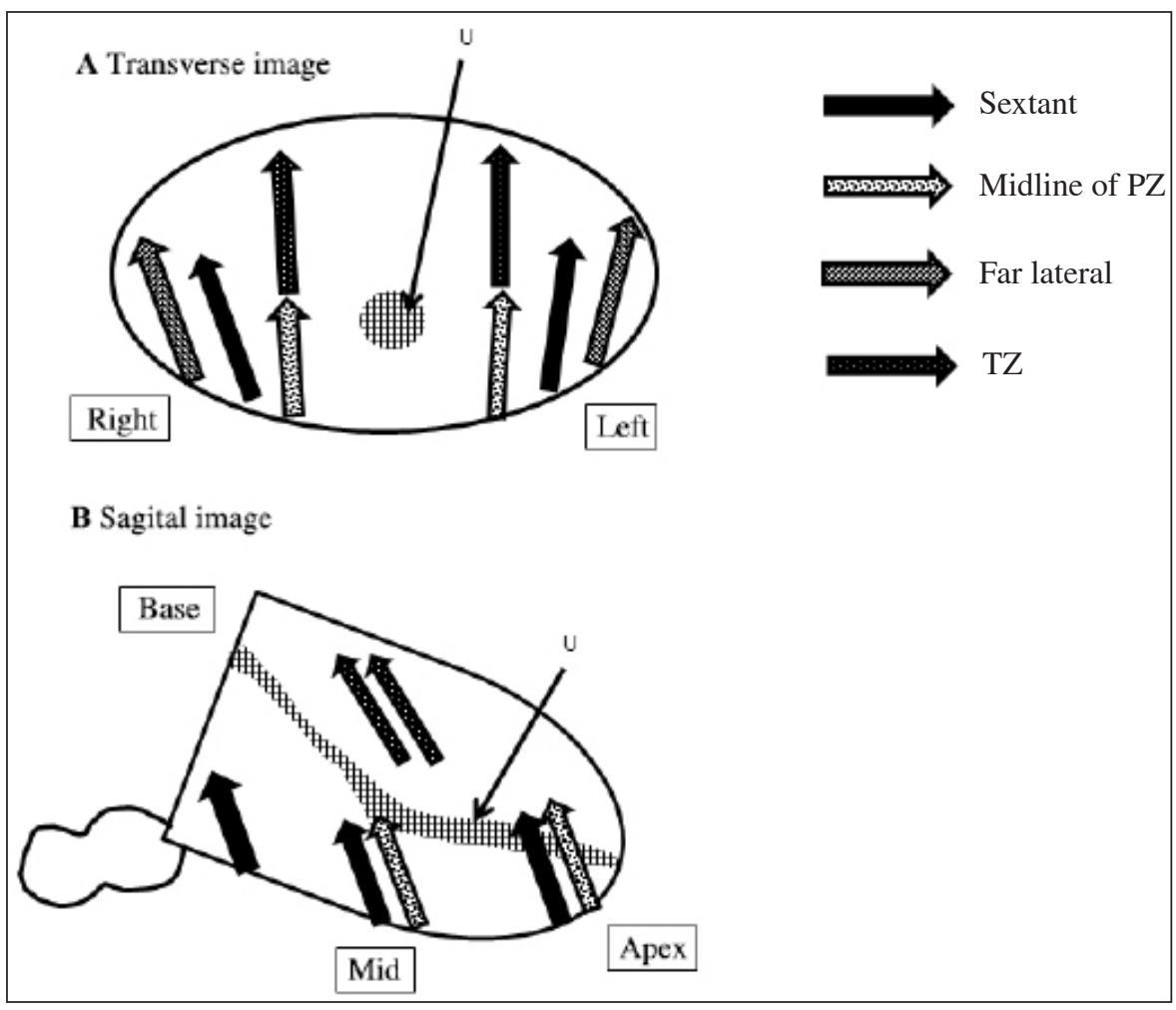

Fig. 1. Schema of endorectal prostate biopsy of transverse image (A) and sagital image (B). $\mathrm{U}=$ Urethra; $\mathrm{PZ}=$ peripheral zone; $\mathrm{TZ}=$ transition zone.

be adapted to Asian populations, and in Japanese people in particular. Furthermore, adapting these protocols for our specific community, despite the similarities in prostate cancer irrespective of the patient's location of origin, may not be appropriate because of possible differences in cancer position depending on ethnicity.

Thus, the initial optimal methods for endorectal prostate biopsy, including core numbers and locations, are uncertain for each ethnicity, and the optimal biopsy method for each specific community require estimation.

This study was designed to estimate the improved accuracy of PCa detection resulting from additional midline biopsies of the peripheral zone over the first standard endorectal sextant pattern in Japanese patients.

\section{Patients and Methods}

Between February 1995 and December 2012, a total of 1,281 patients underwent first endorectal core biopsies due to a suspicion of $\mathrm{PCa}$ as a result of elevated serum prostate specific antigen (PSA) above the normal range ( $>4.0 \mathrm{ng} / \mathrm{ml}$ before biopsy), and were recruited in this retrospect study. This study was approved by our institutional review board and full written informed consent was obtained from all patients.

Midline Biopsies for Prostate Cancer
To elucidate the improvement in detection rate induced by additional midline biopsies, the number of biopsies was increased longitudinally. Patients were classified into 3 groups: 402 cases of sextant biopsies (1995-2002), 488 cases of 8-core biopsies with 2 additional biopsies, consisting of the bilateral midline on mid part (2003-2006), and 391 cases of 10-core biopsies with 4 additional midline biopsies, consisting of bilateral midline at the mid and apex (2007-2012; fig. 1). The prostate volume was calculated based on endorectal ultrasound image before the procedure and an $18 \mathrm{G}$ spring-loaded needle biopsy was used for examination. Prostate specific antigen density (PSAD) was estimated using PSA divided by prostate volume $(\mathrm{ml})$. The biopsy order was performed as follows: the first positions were base, mid, and apex of the right side, the second sites were additional right midline side (mid or mid with apex), the third points were the base, mid, and apex of the left side, and the fourth sites were the additional left midline side (mid or mid with apex). Each biopsy core was separated in the individual container and evaluated.

Variations in Gleason scores (GS) were estimated and reviewed by a single central pathologist. PSA was estimated at the first visit to our institution. The positive rate of each number of biopsies and changes in positive rates associated with PSA ranges were estimated. In addition, GS distribution of positive cases in 10-core biopsy cases, classified by the location of the positive site (sextant only, midline site only, and both sites), were compared.

The Chi-squared and un-paired t-tests were used to compare categorical variables. Differences with $\mathrm{p}$-values $<0.05$ were con- 
Table 1. Baseline characteristics

\begin{tabular}{llll}
\hline Biopsy characteristic & Sextant & 8 Cores & 10 Cores \\
\hline Year term & $1995 / 2-2002 / 10$ & $2002 / 11-2006 / 12$ & $2007 / 1-2012 / 12$ \\
Number & 402 & 488 & 391 \\
Age (range) & $67.8 \pm 6.8(48-89)$ & $67.8 \pm 7.5(36-86)$ & $67.0 \pm 7.5(38-85)$ \\
Prostate volume (ml) mean \pm SD (range) & $32.8 \pm 17.6(4.0-134.4)$ & $35.8 \pm 21.1 *(6.1-234.0)$ & $33.3 \pm 16.4(5.0-52.7)$ \\
PSA (ng/ml) mean \pm SD (range) & $17.0 \pm 38.7(4.1-394.3)$ & $15.6 \pm 30.5(4.0-439.7)$ & $11.5 /-14.7 * *(0.8-130.1)$ \\
PSAD (ng/ml/ml) mean \pm SD (range) & $0.65 \pm 1.50(0.07-17.16)$ & $0.50 \pm 0.71 *(0.04-6.89)$ & $0.42 /-0.61 * *(0.06-6.53)$ \\
PSA (ng/ml) 4.01-10 & 264 & 307 & 273 \\
PSA (ng/ml) 10.01-20 & 79 & 102 & 78 \\
PSA (ng/ml) 20.01- & 59 & 79 & 40 \\
\hline
\end{tabular}

$* \mathrm{p}<0.05, * * \mathrm{p}<0.01$ (vs. sextant).

Table 2. Cancer detection rate on each number of biopsy

\begin{tabular}{|c|c|c|c|}
\hline Number of cancers in total cases $(\%)$ & $123 / 402(30.1)$ & $212 / 488(43.4)^{* *}$ & $208 / 391(53.1)^{* * \dagger \dagger}$ \\
\hline \multicolumn{4}{|c|}{ Number of cancers in each PSA range (\%) } \\
\hline PSA: $10.01-20 \mathrm{ng} / \mathrm{ml}$ & $21 / 79(26.5)$ & $54 / 102(52.9)^{* *}$ & $56 / 78(71.8)^{* * \dagger}$ \\
\hline PSA: $20.01-\mathrm{ng} / \mathrm{ml}$ & $43 / 59(72.9)$ & $66 / 79(83.5)$ & $34 / 40(85.0)$ \\
\hline
\end{tabular}

$* \mathrm{p}<0.05$ or $* * \mathrm{p}<0.001$ (vs. sextant) $\dagger \mathrm{p}<0.05, \dagger \dagger \mathrm{p}<0.01$ or $\dagger \dagger \dagger \mathrm{p}<0.001$ (vs. 8cores).

sidered statistically significant. All statistical analyses were performed using SPSS version 15 software (SPSS Inc, Chicago, IL, USA).

\section{Results}

Baseline characteristics of all cases are shown in table 1. There were no significant differences in the ages or PSA distributions between groups. Even though slightly larger prostate volumes in the 8-cores group and slightly lower serum PSA levels in the 10-cores group were estimated, PSAD significantly decreased longitudinally. The positive rate of core biopsy was significantly improved and was associated with an increased number of cores: $30.1 \%$ for sextant, $43.4 \%$ for 8 -core biopsies $(\mathrm{p}<0.001$ ), and $53.1 \%$ for 10 -core biopsies $(\mathrm{p}<0.001)$. Further, in comparing 8-core biopsies and 10-core biopsies, 10 biopsies significantly improved cancer detection, even though longitudinal decline of PSAD was demonstrated.

The accuracy of biopsies for each PSA range was also significantly improved $(22.3 \%$ for sextant, $30.0 \%$ for 8 -core biopsies [p $<0.05$ ], and $43.2 \%$ for 10 -core biopsies $[\mathrm{p}<0.001]$ in the PSA gray zone $[4.01-10 \mathrm{ng} / \mathrm{ml}]$; and $26.5 \%$ for sextant, $52.9 \%$ for 8 -core biopsies [p < $0.001]$, and $71.8 \%$ for 10 -core biopsies $[p<0.001]$ in the intermediate PSA range [10.1-20 ng/ml]). However, there was no significant difference in the high PSA group (> $20.01 \mathrm{ng} / \mathrm{ml}$ ), yielding positive rates of $72.6 \%$ for sextant, $83.5 \%$ for 8 -core biopsies, and $85.0 \%$ for 10 -core biopsies.

Again comparing 8- and 10-core biopsies, 10-core biopsies significantly improved cancer detection in the PSA gray zone $(4.01-10 \mathrm{ng} / \mathrm{ml} ; \mathrm{p}<0.001)$ and in the intermediate PSA range $(10.1-20 \mathrm{ng} / \mathrm{ml} ; \mathrm{p}<0.05)$.

With respect to the greatest improvement in positive rate, 8 -core biopsies $(52.9 \%)$ reached twice the positive rate of sextant biopsies $(26.5 \%)$ in the PSA range 10.1$20 \mathrm{ng} / \mathrm{ml}$ (table 2).

In the 208 cases with positive results using the 10-core biopsy method, cases were divided into 3 groups based on the positive location, resulting in 129 cases $(62.0 \%)$ for both sites, 47 cases (22.6\%) for sextant only, and 32 cases $(15.4 \%)$ for midline site only. A significantly higher GS was observed in the group with bilateral positive sites (bilateral versus sextant, $\mathrm{p}<0.05$; bilateral versus midline side, $p<0.001)$. GS distribution was not different between the sextant only group and the midline site only group. The relevance of low GS cancer $(\mathrm{GS}=6)$ was not significantly different between the sextant only group $(51.0 \%)$ and the midline only group $(65.6 \%$; table 3$)$. 
Table 3. Distribution of Gleason score based on the location of cancer

\begin{tabular}{llll}
\hline Location of cancer & Bilateral & Sextant & Midline \\
\hline Total number (\%) & $129(62.0)$ & $47(22.6)$ & $32(15.4)$ \\
GS 6, number (\% of each location) & $32(24.8)$ & $24(51.0)^{*}$ & $21(65.6)^{* *}$ \\
GS 7, number (\% of each location) & $59(45.7)$ & $18(38.3)^{*}$ & $7(21.9)^{* *}$ \\
GS 8, number (\% of each location) & $38(51.0)$ & $5(10.6)^{*}$ & $4(12.5)^{* *}$ \\
\hline
\end{tabular}

$* \mathrm{p}<0.05$ : bilateral vs. sextant; $* * \mathrm{p}<0.001$ : bilateral vs. midline site.

\section{Discussion}

The optimal initial method of prostate biopsy, consisting of the number of cores and location, has been recently estimated. According to a review article, 10- to 12-core extended biopsies increases cancer detection compared with the sextant method, and apical and laterally extended sampling of the peripheral zone is recommended [6]. On the contrary, adding a biopsy of the midline part does not improve cancer yield [5]. Furthermore, routine midline peripheral zone biopsy was not recommended due to the lack of enhancement of detection in the first biopsy [8], and, instead, the far lateral peripheral zone should be biopsied. However, these reports were derived from a Western population, and thus the evaluation of the benefit of midline biopsies in Asian populations is uncertain.

According to our data, additional midline first peripheral zone biopsy drastically improved the accuracy of cancer detection in our Japanese population.

With respect to the discrepancy of the benefit of additional midline side biopsies, there is a possibility that ethnic background may influence the distribution of prostate cancer.

In fact, even though prostate biopsy strategies in the West are supported by mapping of prostatectomy specimens $[7,9]$, we do not know much about the difference of anatomical prostate cancer location in men from Western areas and those from Asian areas. According to radiological evaluation of Asian prostates, even though the majority of higher GS and larger tumors with marked enhancement exist in the lateral peripheral zone, the midline of the peripheral zone with lower peak intensity should be treated as suspicious [10], which supports the benefit of a midline biopsy.

Since there is insufficient evidence regarding differences in cancer location based on ethnic background, individual community and ethnic background based studies are indispensable for determining the optimal biopsy method.

Midline Biopsies for Prostate Cancer
In general, the anterior horn was the most frequent positive biopsy site followed by the transition zone and midline [11]. Other studies also report the utility of anterior apex apical core biopsies [12, 13]. In accordance with the aforementioned report, our data also suggest that 10-core biopsies, consisting of the additional apical side with the sextant, resulted in the highest identification of cancer. Since the midline side of the apex biopsy may provide a specimen similar to the anterior apex apical core biopsy, anterior apex apical core biopsies are likely useful for Japanese patients.

Conversely, 8 biopsies consisting of the additional midline of the mid side with the sextant provided a significant improvement in cancer detection, which was not in accordance with a previous report [11]. Thus, in Japanese cases, not only biopsies consisting of the apical midline peripheral zone, but also the additional midline of the mid peripheral zone, could possibly improve the accuracy of detection. To clarify the surprising increased efficacy with the extended midline biopsy, 8-core biopsies consisting of the sextant with the midline at the mid or apical side of the extended biopsy should be compared prospectively in the future.

Previous research has shown that with high PSA $(>20$ $\mathrm{ng} / \mathrm{ml}$ ), an increasing number of core biopsies does not increase the percentage of positive cases [14]. In accordance with this report, our study suggested that 10 biopsies provided a significant improvement in cancer detection in the intermediate PSA range (10.1-20 $\mathrm{ng} / \mathrm{ml})$, but not in the high PSA range $(>20.01 \mathrm{ng} / \mathrm{ml})$. Based on this result, since increased biopsy cores did not contribute to an improvement in cancer detection, six cores may be appropriate for cases with high PSA ( $>20.01 \mathrm{ng} / \mathrm{ml})$. To improve the detection rate for patients with high PSA, another strategy, such as targeted biopsy under magnetic resonance imaging, should be adopted [15].

In general, to obtain a specimen from the transitional zone under rigid endorectal guidance, the tip of the needle needs to be inserted toward the surgical margin of the peripheral zone near the transitional zone. In contrast, in our midline side biopsy, since the tip of needle should be started from the prostate capsule near the rectum, the majority of the specimen is from the peripheral area. However, in cases of benign prostatic hyperplasia, the tip of needle reaches the transition area and the incidence of contamination of the transition zone may increase due to the thin peripheral zone.

Even though the benefit of a transition zone biopsy is uncertain [6], our study might have been affected by the prevalence of contamination with the transition zone. To

Curr Urol 2014;8:74-78 
clarify whether the midline peripheral zone or transition zone contributed to an improvement of cancer detection, the specific cancer location should be estimated in the specimen, and the anterior direction marked with Indian ink.

The most important aim of our research is to increase the detection of significant cancer while minimizing the detection of clinically insignificant cancer (occult and indolent) [6]. In addition, a potential drawback to extended biopsies is the increased tendency of detecting insignificant prostate cancer [6]. Even though the definition of significant or insignificant cancer is clear, we must focus on the distribution of GS in only the additional cores, as it is a factor for clinical risk classification or screening candidates for clinical treatment. According to our data, the distribution of GS was not different between the sextant only group and the midline site only group, and, furthermore, the relevance of low GS cancer (GS
=6) was not significantly different between the sextant only group $(51.0 \%)$ and the midline only group $(65.6 \%)$. Thus our protocol with additional midline site biopsies may not improve the prevalence of cancer detection due to increased insignificant cancer with GS or candidates not suitable for clinical treatment. Although our study is limited due to its longitudinal and retrospective nature, our data strongly suggest that cancer detection can be improved following additional midline peripheral zone biopsies.

In conclusion, midline additional peripheral zone biopsy may be important for improving the accuracy of cancer detection in Japanese populations. In the future, prospective studies that focus on the benefit of midline peripheral zone biopsies should be performed in order to determine the optimal biopsy method available for our Japanese community.

\section{References}

>1 Hodge KK, McNeal JE, Terris MK, Stamey TA: Random systematic versus directed ultrasound guided transrectal core biopsies of the prostate. J Urol 1989;142:71-74.

-2 Presti JC Jr, Chang JJ, Bhargava V, Shinohara $\mathrm{K}$ : The optimal systematic prostate biopsy scheme should include 8 rather than 6 biopsies: results of a prospective clinical trial. $\mathrm{J}$ Urol 2000;163:163-166.

>3 Durkan GC, Sheikh N, Johnson P, Hildreth AJ, Greene DR: Improving prostate cancer detection with an extended-core transrectal ultrasonography-guided prostate biopsy protocol. BJU Int 2002;89:33-39.

$\checkmark 4$ Taylor JA 3rd, Gancarczyk KJ, Fant GV, McLeod DG: Increasing the number of core samples taken at prostate needle biopsy enhances the detection of clinically significant prostate cancer. Urology 2002;60:841-845.

$\checkmark 5$ Eichler K, Hempel S, Wilby J, Myers L, Bachmann LM, Kleijnen J: Diagnostic value of systematic biopsy methods in the investigation of prostate cancer: a systematic review. J Urol 2006;175:1605-1612.
-6 Bjurlin MA, Carter HB, Schellhammer P, Cookson MS, Gomella LG, Troyer D, Wheeler TM, Schlossberg S, Penson DF, Taneja SS: Optimization of initial prostate biopsy in clinical practice: sampling, labeling and specimen processing. J Urol 2013;189: 2039-2046.

$\checkmark 7$ Chen ME, Johnston DA, Tang K, Babaian RJ, Troncoso P: Detailed mapping of prostate carcinoma foci: biopsy strategy implications. Cancer 2000;89:1800-1809.

>8 Guichard G, Larre S, Gallina A, Lazar A, Faucon H, Chemama S, Allory Y, Patard JJ, Vordos D, Hoznek A, Yiou R, Salomon L, Abbou C C, de la Taille A: Extended 21-sample needle biopsy protocol for diagnosis of prostate cancer in 1000 consecutive patients. Eur Urol 2007;52:430-435.

$>9$ McNeal JE: Cancer volume and site of origin of adenocarcinoma in the prostate: relationship to local and distant spread. Hum Pathol 1992;23:258-266.

10 Jiang J, Chen YQ, Zhu YK, Yao XH, Qi J: Factors influencing the degree of enhancement of prostate cancer on contrast-enhanced transrectal ultrasonography: correlation with biopsy and radical prostatectomy specimens. Brit J Radil 2012;85:e979-986.
11 Babaian RJ, Toi A, Kamoi K, Troncoso P, Sweet J, Evans R, Johnston D, Chen M: A comparative analysis of sextant and an extended 11-core multisite directed biopsy strategy. J Urol 2000;163:152-157.

$>12$ Meng MV, Franks JH, Presti JC Jr, Shinohara $\mathrm{K}$ : The utility of apical anterior horn biopsies in prostate cancer detection. Urol Oncol 2003;21:361-365.

$>13$ Moussa AS, Meshref A, Schoenfield L, Masoud A, Abdel-Rahman S, Li J, Flazoura S, Magi-Galluzzi C, Fergany A, Fareed K, Jones JS: Importance of additional "extreme" anterior apical needle biopsies in the initial detection of prostate cancer. Urology 2010;75: 1034-1039.

$>14$ Presti JC Jr, O’Dowd GJ, Miller MC, Mattu R, Veltri RW: Extended peripheral zone biopsy schemes increase cancer detection rates and minimize variance in prostate specific antigen and age related cancer rates: results of a community multi-practice study. J Urol 2003;169:125-129.

15 Moore CM, Robertson NL, Arsanious N, Middleton T, Villers A, Klotz L, Taneja SS, Emberton M: Image-guided prostate biopsy using magnetic resonance imaging-derived targets: a systematic review. Eur Urol 2013; $63: 125-140$ 